\title{
Fuzzy Based Design for Third-Party Pipeline Failures in the Niger Delta Region of Nigeria
}

\section{*11ARIAVIE, GO; EMETITIRI, RE; ONONEME, OE; OLOWU, ID}

Mechanical Engineering Department, University of Benin, Benin City, Nigeria

ariaviefe@uniben.edu; 2348035615205

\begin{abstract}
A Fuzzy based design for assessing failures of pipelines occasioned by third party in the Niger Delta Region of Nigeria has been proposed in this study. Twelve factors earlier identified by Ariavie et al (2010), were further grouped into four, viz : violence, desperation, misconduct and agricultural categories to allow for a less tedious rule base development. Each category has inputs (base inputs) plugged into it which is in turn plugged into another system (main engine). Based on this, the fuzzy model was designed, using MATLAB fuzzy toolbox to develop a hypothetical simulation which simply involves the feeding of randomly generated values between 0 and 1 to the controllers. The evaluation process of the first expert is presented and obtained for the four categories Risk values as $0.533,0.509,0.442$ and 0.552 for violence, desperation, misconduct and agricultural respectively, with total risk value being 0.063 . $\odot$ JASEM
\end{abstract}

\section{http://dx.doi.org/10.4314/jasem.v19i2.9}

KEYWORDS: Fuzzy logic design; Third-party pipeline failures; Risk analysis

Introduction

Third-party pipeline failures are occasioned by interference of outside or external forces to the pipeline owners or pipeline itself due to encroachment into the right-of-way of pipelines (Ariavie et al, 2010, Ariavie et al 2011 and Ariavie and Ovuworie, 2012). Third-party risk affects the extent and possibility of harm or loss to humans and pipeline operators (in the form of personal injury or death, environmental degradation, property damage, loss or destruction of facilities). Consequently, it becomes imperative to design against pipeline failures that emanate from third-party activities, so as to eliminate or minimize them as much as possible.

Analysis procedures for third-party pipeline failures have so far been statistical in nature. However, requisite data are sparsely available in most developing countries, including Nigeria hindering mitigation actions that are essential for planning and enhanced decision making. Hence, an alternative that would require less mathematical formulation and rigor becomes imperative and therefore, fuzzy logic comes in handy (Novak et al, 1999). Due to its advantages and wide applicability, fuzzy logic method has gained much development and is discussed in detail as a subject in standard literature (Yager and Filer, 1994; Sivanandam, Sumathi and Deepa, 2001; Novak and Mockr, 1999). Fuzzy logic has become handy and it works well for addressing poorly characterized parameters and linguistic variables (Darbra et al., 2008).

\section{MATERIALS AND METHOD}

The twelve factors identified by Ariavie et al. (2010) as those that affect pipeline safety were adopted in this study. Because of tedious computations, the twelve factors were grouped into four (Table 1) categories allowing for a less tedious rule base development. Each category input (base input) is plugged into another system (main engine) as shown in Figure 1generating a single value at output (defuzzification), which is a fuzzy score assigned to that category. This score in turn is further plugged into the main fuzzy system which gives the risk evaluation value of the studied demographic region. The number of input variables and their associated membership functions determine the number of rules which oftentimes can reflect experiences of the human experts. Each rule reflects a non-linear relationship between independent variables (inputs) and dependent variables (outputs) of the process or system under consideration.

The identified steps taken in the design would include: 1. Identification of the input and output variables (the system's variables), 2. Determination of the ranges of input and output variables, 3 . Determination of the membership functions for selected input and output variables, 4. Formation of the set of linguistic rules that represent the 
relationships between the system variables, 5 . Selection of the appropriate reasoning mechanism to the formalization of the Fuzzy model (Mamdani inference technique), and; 6. Defuzzification.

Table 1: Category Arrangements of Hazardous Factors

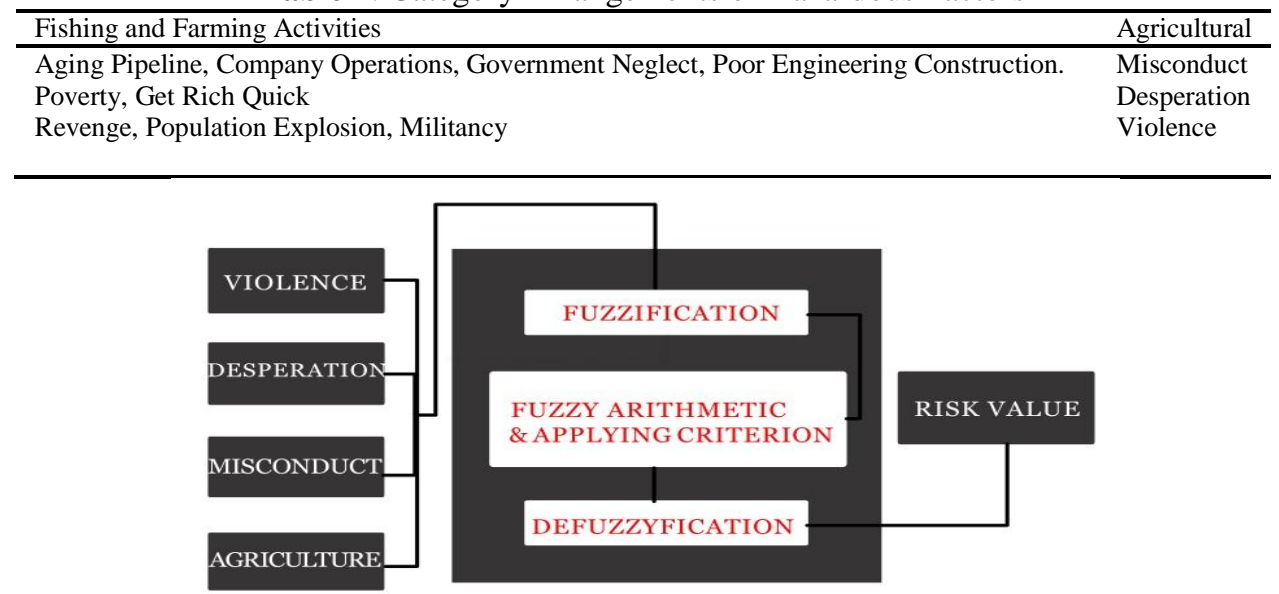

Fig 1: Category Input Layout for Total Risk Factor

Designs for Agricultural Risk Factors: Two factors of Farming and Fishing were combined as input data to give an output variable of Agriculture risk factor. The process of Fuzzification involves the assignment of membership function to the selected input as shown

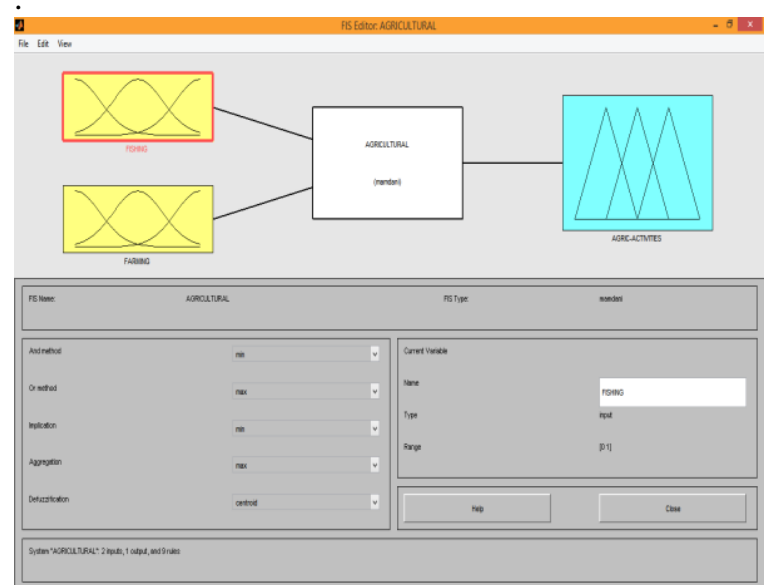

Fig 2: Agricultural Factor Sub Fuzzy System Process Diagram.

The figures above represents a 3 - grade system of ; Low, Medium and high with corresponding range values of $(0-0.4,0.2-0.8$, and $0.6-1)$ and by defining in figure 2, using three steps grading system for the input variable and five for the output variable. The input and output is modeled with the adoption of the triangular membership function profile on a homogenous scale

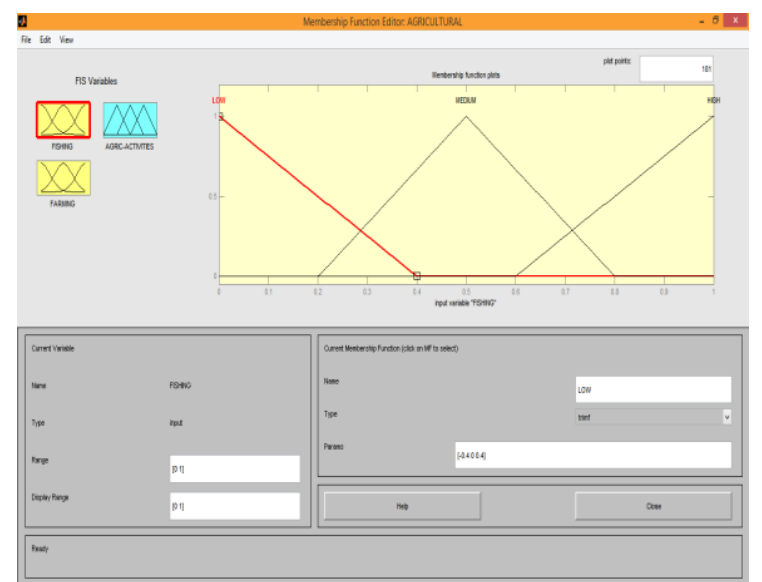

Table 2: Rule Table for the Agricultural Risk Sub-Fuzzy Engine

$\frac{T}{6}$

\begin{tabular}{|c|c|c|c|c|c|c|c|c|c|c|c|c|}
\hline 1 & If & Farming & Is & Low & And & Fishing & Is & Low & Is & Agric risk & Is & Very low \\
\hline 2 & If & Farming & Is & Low & And & Fishing & Is & Medium & Is & Agric risk & Is & Low \\
\hline 3 & If & Farming & Is & Low & And & Fishing & Is & High & Is & Agric risk & Is & Medium \\
\hline 4 & If & Farming & Is & Medium & And & Fishing & Is & Low & Is & Agric risk & Is & Low \\
\hline
\end{tabular}




\begin{tabular}{|c|c|c|c|c|c|c|c|c|c|c|c|c|}
\hline 5 & $\overline{\text { If }}$ & Farming & Is & Medium & And & Fishing & Is & Medium & Is & Agric risk & Is & Medium \\
\hline 6 & If & Farming & Is & Medium & And & Fishing & Is & High & Is & Agric risk & Is & High \\
\hline 7 & If & Farming & Is & High & And & Fishing & Is & Low & Is & Agric risk & Is & Medium \\
\hline 8 & If & Farming & Is & High & And & Fishing & Is & Medium & Is & Agric risk & Is & High \\
\hline 9 & If & Farming & Is & High & And & Fishing & Is & High & Is & Agric risk & Is & Very high \\
\hline
\end{tabular}

The next step defuzzifies the output membership plot by the center of gravity method yielding an output value based on expert input of the agricultural risk factor, and this is related with other category sub fuzzy engine outputs. This design process is continued for the remaining categories using the same input membership function profile. In Simulink of MATLAB, parameters and states are represented by blocks, while signals are represented by the lines that connect blocks. For each subsystem identified, two wo input signals for the Agricultural and Desperation Risk subsystem, five for the misconduct/attack subsystem and three for the

violence risk subsystem and one output signals which represents the total risk.

The fourth step: Building the Simulink Block Diagram

This step is extensively the most consuming part of the design. It involves a careful selection of blocks with attributes enough to perform desired function. Careful thinking has been incorporated into designing this fuzzy Simulink system. Figure 4 is an adopted image of the Simulink model for Agricultural and Misconduct/Attack Risk Factors in Matlab.
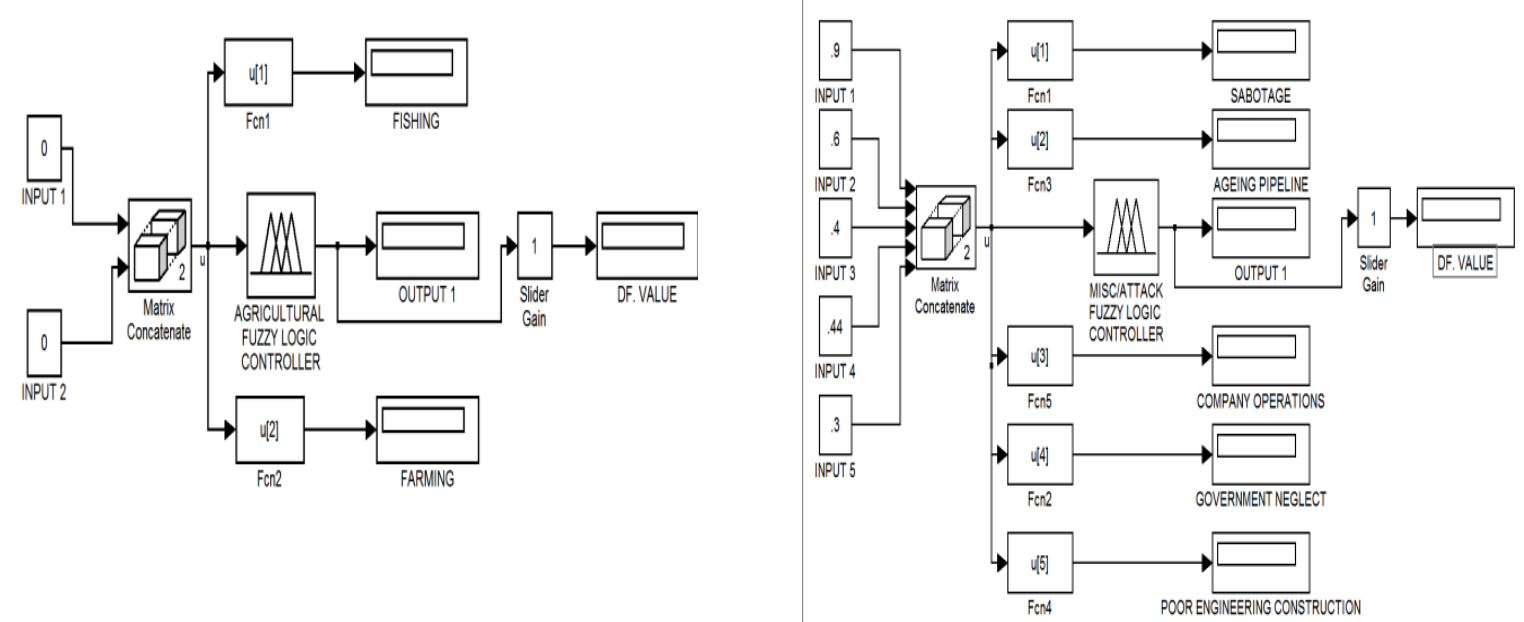

Fig 4: Working Simulink Model for Agricultural and Misconduct/Attack Risk Factors

The fifth step: Running the Simulation: After building the Simulink block diagram, next is to simulate the model and analyze the results. Simulink allows for defining interactive system inputs, simulate the model, and observe changes in behavior. This allows you to quickly evaluate your model.

The sixth step: Validating the Simulation Results: Finally, validate the model accurately and confirm that it represents the physical characteristics of the dynamic system.

With the completion of system design, the next step involves testing to determining whether the system is viable and can be trusted, but before the system is fed with various inputs, the mode of operation needs to be explained. The working process involves a manual feeding of values which are defined by experts opinions, these values can be obtained by utilizing the vivid flexibilities of questionnaires. The basic elements of the questionnaire should include the inputs and a way to pass judgment in relation to their effects on pipeline safety

A scale sample and question could be represented as follows

Question 1: Do you think human poverty has an effect on pipeline safety? Yes No

If yes, to what extent do you think it affects pipeline safety? Mark the scale to record your opinion.

0 100

Low

Medium

High 
This is the method by which expert opinion are obtained for a total of 12 inputs, the values are to be precisely read off the scale by measuring the length from the zero point position and interpolating to find the corresponding value with $\mathrm{NO}$ being of zero value.

The next step is that of evaluation, a process whence the already modeled system is fed with input in their converted form since the fuzzy scale has a support between 0 and 1 it is therefore necessary to convert the values obtained from percentage to decimal.
Hypothetical Simulation: For a comprehensive understanding of how the system works, an example is vital and for this reason a hypothetic simulation is provided.

The simulation simply involves the feeding of randomly generated values between 0 and 1 to the controllers and ascertaining the risk involved with each combination for these example, four data sets are created representing data inputs from hypothetical experts in its already converted form, this answers are listed in a Table 3

Table 3: Hypothetical Data for Simulation

\begin{tabular}{lllll}
\hline & \multicolumn{3}{c}{ Agricultural } \\
\hline Data/input & Expert 1 & Expert 2 & Expert 3 & Expert 4 \\
Fishing & 0.7 & 0 & 0 & 0.3 \\
Farming activities & 0.4 & 0.2 & 0.1 & 0.4 \\
Misconduct/attack & & & & \\
Data/input & Expert 1 & Expert 2 & Expert 3 & Expert 4 \\
Sabotage & 0.5 & 0.8 & 0.75 & 0.9 \\
Aging piprline & 0.9 & 0.3 & 0.7 & 0.6 \\
Company operation & 0.7 & 0.45 & 0.88 & 0.65 \\
Government neglect & 0.2 & 0.5 & 0.62 & 0.9 \\
Poor engineering & 0.81 & 0.7 & 0.64 & 0.5 \\
Construction & & & & \\
Desperation & & & & \\
Data/input & Expert 1 & Expert 2 & Expert 3 & Expert 4 \\
Poverty & 0.43 & 0.8 & 0.7 & 0.65 \\
Get rich quickly & 0.89 & 0.4 & 0.5 & 0.65 \\
Violence & & & & \\
Data/input & Expert 1 & Expert 2 & Expert 3 & Expert 4 \\
Revenge & 0.5 & 0.8 & 0 & 0.7 \\
Population explosion & 0.2 & 0.3 & 0.5 & 0.3 \\
Militancy & 0.8 & 0.7 & 0.7 & 0.5 \\
\hline
\end{tabular}

With these generated values, evaluation is possible; simply by changing the value at the input nodes to the systems, the risk value can be ascertained. A detailed process on how the first expert data is evaluated will be discussed in the next section, only risk values for the other experts will be presented, bear in mind that they follow the same method of evaluation.

First stage of evaluation: Risk evaluation is carried out by the four models, effected by changing the inputs and running the simulation after which a single value is obtained at the output, each value obtained represents the risk of the factor on the pipelines.
Second stage of evaluation: This stage is almost similar to the first only that the values used here are from the subsystems simulated earlier, the four categories make up the input to this system, as shown in table 4 these category have the headings: Agricultural Risk, Misconduct/Attack Risk, Desperation Risk And Violence Risk.

The routine is re-run using these new values derived from the evaluation of expert 1 data set, and the results of all the experts are given in table 5 
Table 4: Evaluated Opinion Result

\begin{tabular}{lllll}
\hline & \multicolumn{3}{c}{ AGRICULTURAL } \\
\hline Data/Input & EXPERT 1 & EXPERT 2 & EXPERT 3 & EXPERT 4 \\
Fishing & 0.7 & 0 & 0 & 0.3 \\
Farming Activities & 0.4 & 0.2 & 0.1 & 0.4 \\
RISK & 0.5879 & 0.1551 & 0.0800 & 0.4116 \\
Misconduct/Attack & & & & \\
Data/Input & EXPERT 1 & EXPERT 2 & EXPERT 3 & EXPERT 4 \\
Sabotage & 0.5 & 0.8 & 0.75 & 0.9 \\
Aging Piprline & 0.9 & 0.3 & 0.7 & 0.6 \\
Company Operation & 0.7 & 0.45 & 0.88 & 0.65 \\
Government neglect & 0.2 & 0.5 & 0.62 & 0.9 \\
Poor engineering & 0.81 & 0.7 & 0.64 & 0.5 \\
Construction & & & & \\
RISK & 0.5426 & 0.5574 & 0.7293 & 0.7204 \\
Desperation & & & & \\
Data/input & EXPERT 1 & EXPERT 2 & EXPERT 3 & EXPERT 4 \\
Poverty & 0.43 & 0.8 & 0.7 & 0.65 \\
Get rich quickly & 0.89 & 0.4 & 0.5 & 0.65 \\
Risk & 0.75 & 0.7491 & 0.5879 & 0.5725 \\
Violence & & & & \\
Data/input & EXPERT 1 & EXPERT 2 & EXPERT 3 & EXPERT 4 \\
Revenge & 0.5 & 0.8 & 0 & 0.7 \\
Population explosion & 0.2 & 0.3 & 0.5 & 0.3 \\
Militancy & 0.8 & 0.7 & 0.7 & 0.5 \\
Risk & 0.5 & 0.6188 & 0.3806 & 0.5 \\
\hline
\end{tabular}

Table 5: Subsystem Output

\begin{tabular}{|l|l|l|l|l|}
\hline DATA / INPUT & EXPERT 1 & EXPERT 2 & EXPERT 3 & EXPERT 4 \\
\hline AGRICULTURAL RISK & 0.5879 & 0.1551 & 0.0800 & 0.4116 \\
\hline MISC./ATTACK RISK & 0.5426 & 0.5574 & 0.7293 & 0.7204 \\
\hline DESPERATION RISK & 0.75 & 0.7491 & 0.5879 & 0.5725 \\
\hline VIOLENCE RISK & 0.5 & 0.6188 & 0.3806 & 0.5 \\
\hline TOTAL RISK & 0.533 & 0.509 & 0.442 & 0.522 \\
\hline
\end{tabular}

\section{RESULT AND DISCUSSION}

This section explains the method by which the various risks are combined. A proposed system is to find the mean value of the total risks to produce a single value, taking into consideration every experts opinion. This method would be used for the hypothetical simulation carried out. The mean value is computed for the above as follows using probabilistic theory of mutuality:

Expert1's opinion amounted to a risk value of 0.533 ; Expert2's opinion amounted to a risk value of 0.509
Expert3's opinion amounted to a risk value of 0.442 ; Expert4's opinion amounted to a risk value of 0.522 The total risk value for this hypothetical session $=$ $(0.533 \times 0.509 \times 0.442 \times 0.522)=0.063$

This value obtained gives a sense of how risky an area is under the scope of assessment.

In order to use the developed model to assess the risk of pipelines failure, the different information and characteristics of the network pipelines can be collected and used as input data to the model. The output of the model is a number that represents the risk of failure of each pipeline and can be represented on a scale (0-10) as shown in figure 5.

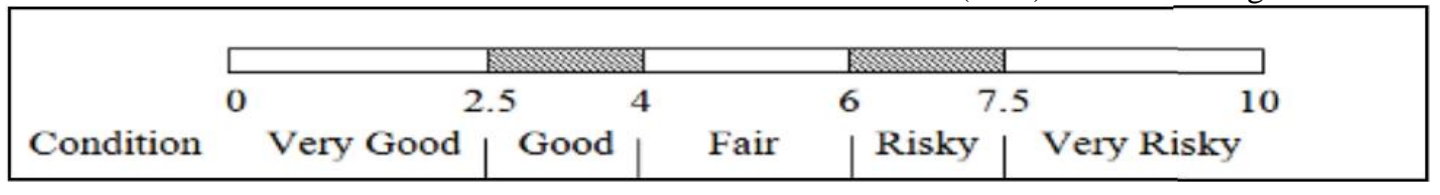

*IARIAVIE, GO; EMETITIRI, RE; ONONEME, RE; OLOWU, ID 
Fig 5: Proposed Risk of Failure Scale (Fares et al, 2009)

For the assessment session above which yielded a value of 0.063 in the premise of 0 and 1 , it is only logical to multiply this value by 10 to use this proposed scale.

Conclusion: In this paper, a fuzzy based design methodology has been proposed for evaluating the risk of oil transport pipelines due to third-party activities. Applicability of the proposed methodology has been tested on a real case in another field. Findings of the case study demonstrated that the proposed methodology can be easily applied by the professionals to quantify risk ratings. An advantage of the proposed methodology is that it will give investors a more rational basis on which to make decisions and it can prevent cost and schedule overruns. Forecasting the measure of risk of a pipeline can be made by any decision maker with the help of the fuzzy rating tool described in this paper, using Fuzzy Logic Toolbox and Simulink. Various risks that endanger complex systems have very difficult mechanisms of activation so precise mathematical modeling of the system is usually too complex for practical application. Fuzzy logic with its built-in toleration to imprecise data is an ideal tool for enhancing the effectiveness of pipeline risk analysis. It is also worth noting that fuzzy logic approach requires real experiences of experts and competent personnel to identify and collect needed data and to build a fuzzy system. However, subjective evaluation is limited especially when compared to other methods.

\section{REFERENCES}

Ariavie G.O, Ovuworie G.C and Ariavie S.S (2010) "On Expert Elicitation Method in the Determination of Transmission Pipeline Failure occasioned by Third Party Activities in the Niger Delta region of Nigeria".

Proceedings of the International Conference on Engineering Research and Development (ICER\&D 2010) held at the University of Benin, Nigeria. 7th -9th September, 2010.

Ariavie G.O, Ovuworie G.C and Ariavie S.S (2011) "Fuzzy Failure Probability of Transmission Pipelines in the Niger Delta Region of Nigeria: The case of Third Party Activities".Journal of the Nigerian Association of Mathematical Physics. Volume 18. (May 2011), pp 445 - 450
Ariavie G.O and Ovuworie G. C (2012) "Delphi Fuzzy Ellicitation Technique in the Determination of Third Party Failure Probability of Onshore Transmission Pipelines in the Niger Delta Region of Nigeria". J. Appl. Sci. Environ. Manage. March 2012. Vol. 16 (1) 5-10

Bajaj, BK. (2007), "Risk Assessment and Consequence Analysis" Pipeline Projects in Area of Oil. Envirotech Consultants Pvt. Ltd.

David, M. (2007), "Matlab Demystified", The McGraw-Hill Companies

Da Ruan. (2000), "Fuzzy Sets and Fuzzy Information" Key Selected Papers by Lofti A. Zadeh. Beijing.

DECC, (2009) "Pollution Prevention and Oil Spills."

https://www.og.berr.gov.uk/information/bb updates/ chapters/Table chart3 1.htm

Darbra, R.M, Eljarrat E., and Barcelo D (2008). "How to Measure Uncertainties in Environmental Risk Assessment," Trends in Analytical Chemistry, Vol. 27, No. 4, PP 377 385.

Isabel, L., and Mario, S.M (2012). "Applications of Fuzzy Logic in Risk Assessment - The RA_X Case"

Haimen, K. (2009), "Risk Assessment, Modeling and Management", 3rd edition, A John Wiley \& Sons Inc. publication

Jang, R., Gulley, N. (2008). "Fuzzy Logic Toolbox User's Guide" Natick, Massachusetts: The Math Works, Inc.

Landoll, D. (2006). “The Security Risk Assessment Handbook," New York: Auer Bach Publication. Page 473.

Mohammad, F. A, (2012), "Fuzzy Inference System Theory and Applications", Available from:http://www.intechopen.com/books/fuzzyinference-system-theory-andapplications/applications-of-fuzzy-logic-in-riskassessment-the-ra- $\mathrm{X}$-case 
Mendel, J. (2001) -Uncertainty in Fuzzy Logic Systems, Python Developer's handbook

Novak V. Perfilieva and Mockr J. K (1999), "Mathematical Principles of Fuzzy Logic", Academic Press

Pokorádi, L (2009). "Risk Assessment Based upon Fuzzy Set Theory". Procedure of the $15^{\text {th }}$ International Conference of "Building Services, Mechanical Engineering and Building Industry Days", 15-16 October 2009,Debrecen, Hungary, pp. 311-318.

Rudra, P., (2010). "Getting started with MATLAB: A Quick Introduction for Scientists and Engineers". Oxford University Press
Wang, P. (2007), Da Ruan, Kerre E, "Fuzzy Logic a Spectrum of Theoretical \& Practical Issues," page 464

Sivanandam S. N., S. Sumathi and S. N. Deepa (2001), "Introduction to Fuzzy Logic using MATLAB", Berlin Heidelberg, Springer, New York

Yager R. R. and Filer D. P (1994), "Essentials of Fuzzy Modeling and Control”, John Wiley, New York.

Zadeh, L. A. (1978). "Fuzzy Set Theory as the basis for Possibility Theory. Fuzzy Sets and Systems 1 (1978) 3-28. 